\title{
Effects of Tianeptine on Adult Rats Following Prenatal Stress
}

\author{
Hwayoung Lee', Hyung-Ki Kim', Jun-Tack Kwon', Young Ock Kim², Jonghoon Seo', Sanghyun Lee', \\ lk-Hyun Cho ${ }^{4}$, Hak-Jae Kim ${ }^{1}$ \\ ${ }^{1}$ Department of Clinical Pharmacology and Soonchunhyang Medical Research Institute, Soonchunhyang University College of Medicine, \\ Cheonan, ${ }^{2}$ Department of Development of Ginseng and Medical Plants Research Institute, Rural Administration, Eumseong, ${ }^{3}$ Department of \\ Integrative Plant Science, Chung-Ang University, Anseong, ${ }^{4}$ Department of Convergence Medical Science, Brain Korea 21 Plus Program, and \\ Institute of Korean Medicine, College of Oriental Medicine, Kyung Hee University, Seoul, Korea
}

\begin{abstract}
Objective: Exposing a pregnant female to stress during the critical period of embryonic fetal brain development increases the risk of psychiatric disorders in the offspring. The objective of this study was to investigate the effect of antidepressant tianeptine on prenatally stressed (PNS) rats.

Methods: In this study, a repeated variable stress paradigm was applied to pregnant rats during the last week of gestation. To investigate the effects of antidepressant tianeptine on PNS rats, behavioral and protein expression analyses were performed. Forced swim test, open field test, and social interaction test were performed to determine changes in PNS rats compared to non-stressed offspring. Haloperidol was used as a positive control as an antipsychotic drug based on previous studies.

Results: Behavioral changes were restored after treatment with tianeptine or haloperidol. Western blot and immunohistochemical analyses of the prefrontal cortex revealed downregulation of several neurodevelopmental proteins in PNS rats. After treatment with tianeptine or haloperidol, their expression levels were increased.

Conclusion: Downregulation of several proteins in PNS rats might have caused subsequent behavioral changes in PNS rats. After tianeptine or haloperidol treatment, behavioral changes in PNS rats were restored. Therefore, tianeptine might decrease incidence of prenatal stress related-psychiatric disorders such as depression and schizophrenia.
\end{abstract}

KEY WORDS: Prenatal stress; Laboratory animal model; Tianeptine; Haloperidol; Behavior test; Psychiatric disorder.

\section{INTRODUCTION}

Several animal studies have demonstrated that prenatal stress (PNS) such as elevated glucocorticoid level during gestation is associated with biochemical, physiological, and behavioral changes in the offspring, including reduced birth weight, cardiovascular and neuroendocrinological abnormalities, attention dysfunctions, enhanced anxiety-related behaviors, and cognitive deficits. ${ }^{1-10)}$ Pregnant rats are commonly exposed to stressful manipulations during the third week of pregnancy which is ap-

Received: March 27, 2017 / Revised: May 1, 2017

Accepted: May 25, 2017

Address for correspondence: Hak-Jae Kim, PhD

Department of Clinical Pharmacology, Soonchunhyang

University College of Medicine, 31 Suncheonhyang 6-gil,

Dongnam-gu, Cheonan 31151, Korea.

Tel: +82-41-570-2478, Fax: +82-41-575-2406

E-mail: hak3962@sch.ac.kr

ORCID: https://orcid.org/0000-0003-1212-7861 proximately equivalent to the second trimester of human gestation in terms of neural development. ${ }^{2,8-10)}$ Early life exposure to PNS causes neural developmental deficits with decreased number of hippocampal synapses and neurons as well as changed dendritic length and spine density. These deficits are different from those of non-stressed (NS) controls. ${ }^{11,12)}$ Increased corticosterone secretion prolonged by PNS stimulation is associated with decreased levels of corticosterone receptors in the brain. On the other hand, the amplitude and duration of stress-induced corticosterone secretion can be reduced by better postnatal handling such as short periodic maternal deprivation and early adoption in adult offspring. ${ }^{13,14)}$ PNS or maternal exposure to glucocorticoids may affect the responsivity of the hypothalamic-pituitary-adrenal (HPA) axis. It can induce cognitive deficits in offspring. ${ }^{2,8,9)}$ Cognitive deficits resulting from disrupted hippocampal anatomy and impaired function of the HPA axis are also observed in patients with schizophrenia. ${ }^{7)}$ PNS can also

(ㄷ) This is an Open-Access article distributed under the terms of the Creative Commons Attribution Non-Commercial License (http://creativecommons.org/licenses/by-nc/4.0) which permits unrestricted non-commercial use, distribution, and reproduction in any medium, provided the original work is properly cited. 
result in various gene expression changes in rat brain, including genes associated with neural development, cell differentiation, and neurotransmitter function. ${ }^{8,15,16)}$ We have previously reported that dihydropyrimidinase-like 2 (Dpys/2) and LIM and SH protein 1 (Lasp1) are downregulated in the prefrontal cortex of PNS rats. ${ }^{177}$ We have also found that DPYSL2 gene polymorphisms in human might be associated with the development of schizophrenia. ${ }^{17)}$

Cyclic adenosine monophosphate (cAMP) response element-binding protein is a transcription factor with well-established role in learning-related synaptic plasticity. ${ }^{18)}$ It has been reported that prolonged administration of serotonin and/or noradrenaline reuptake inhibitors can enhance the expression and phosphorylation of CAMP response element-binding protein. ${ }^{19-21)}$ Long-term pharmacotherapy can also attenuate stress-induced dendritic reorganization $^{22)}$ and impairment in hippocampal neurogenesis. ${ }^{23,24)}$ In this context, antidepressant tianeptine can improve stress-induced morphological changes in brain areas such as the hippocampus, prefrontal cortex, and amygdala. ${ }^{25)}$ Tianeptine can also trigger a cascade of neuronal adaptations, including increased phosphorylation of N-methyl-D-aspartate (NMDA) and $\alpha$-amino-3-hydroxy-5-methyl-4-isoxazolepropionic acid (AMPA) among different subtypes of glutamate receptors. ${ }^{26)}$ Haloperidol is an antipsychotic drug that affects the glutamatergic system. It can change the expression level of two main subunits or neuro-receptors of metabotropic and ionotropic glutamate receptors. ${ }^{27,28)}$ Glutamate is the predominant neurotransmitter controlling neuronal excitability and neuroplasticity in majority of brain circuits. Glutamatergic dysfunctions have been documented in patients with depression. ${ }^{29,30)}$ It has been reported that tianeptine might be able to attenuate impaired neuroplasticity by modulating the glutamatergic system in key brain structures, including medial prefrontal cortex, amygdala, and hippocampus. ${ }^{31,32)}$ In addition, ttianeptine treatment can induce antidepressive-like effects in rats. ${ }^{33)}$

These findings led us to hypothesize that tianeptine treatment might affect the pathophysiology of PNS-related psychiatric disorders such as depression and schizophrenia. In the present study, offspring behavioral patterns and changes in protein levels were examined in the prefrontal cortex of PNS rats. The objective of this study was to determine whether changes caused by PNS might be affected by tianeptine treatment.

\section{METHODS}

\section{Drugs and Animals}

Tianeptine and haloperidol (Sigma Aldrich, St. Louis, MO, USA; Merck KGaA, Darmstadt, Germany) were dissolved in $0.9 \% \mathrm{NaCl}$. Separate groups of adolescent rats were treated with tianeptine $(10 \mathrm{mg} / \mathrm{kg})$ or haloperidol $(1.5 \mathrm{mg} / \mathrm{kg})^{34,35)}$ once daily for 21 consecutive days (postnatal day 35 to 56 ). ${ }^{10)}$ The duration of experimental manipulation used for rats (P35-P49) was selected because it roughly approximated mid-adolescence in human. ${ }^{36)}$ Each drug treatment was performed at 10 a.m. every day.

\section{PNS Procedures}

Pregnant Sprague-Dawley rats ( $\mathrm{n}=6$ per group) were purchased from Central Lab Animal, Inc. (Seoul, Korea). They arrived at the animal facility on day 7 of gestation. These rats were housed under standard conditions under a 12/12-hour light/dark cycle (lights on at 06:30). They were provided free access to food and water. All animal procedures were performed in accordance with guidelines for the care and use of laboratory animals of the United States National Institutes of Health. ${ }^{37)}$ Animal study protocol used in this study was approved by the Institutional of Animal Care and Use Committee of Soonchunhyang University, Korea (approval No.: $\mathrm{SCH} 16-0058)$. The PNS rat model was prepared as described previously. ${ }^{17,38)}$ PNS exposure was initiated on day 14 of gestation. It included the following: 1) 1-hour restraint in a well-ventilated cylindrical Plexiglas restraint, 2) 6-hour exposure to a cold environment $\left(4^{\circ} \mathrm{C}\right), 3$ ) overnight fasting, 4) 15 minutes of swimming stress in room-temperature water, 5) reversal of the light-dark cy-

Table 1. Prenatal stress paradigm

\begin{tabular}{|c|c|c|c|}
\hline \multirow{2}{*}{$\begin{array}{c}\text { Gestation } \\
\text { day }\end{array}$} & \multicolumn{3}{|c|}{ Time of day } \\
\hline & a.m. & Mid-day & p.m. \\
\hline 14 & Restraint 60 min & Swim 15 min & Restraint 60 min \\
\hline 15 & Cold stress $6 \mathrm{hr}$ & - & Fast overnight \\
\hline 16 & Swim 15 min & Restraint $60 \mathrm{~min}$ & Swim $15 \mathrm{~min}$ \\
\hline 17 & - & Swim 15 min & Light on overnight \\
\hline 18 & Social stress $12 \mathrm{hr}$ & & \\
\hline 19 & Restraint 60 min & Swim 15 min & Restraint 60 min \\
\hline 20 & & Cold stress $6 \mathrm{hr}$ & \\
\hline 21 & Swim 15 min & Restraint $60 \mathrm{~min}$ & Swim 15 min \\
\hline
\end{tabular}


cle, and/or 6) social stress induced by overcrowded housing conditions during the dark phase of the cycle (Table 1). ${ }^{7,8)}$ Pregnant dams used as controls remained in the animal room during gestational days 14 to 21 . They were only exposed to normal animal room husbandry procedures. Following birth, rats and their pups were left undisturbed in their cages until weaning on postnatal day 23. At this time, male and female offspring were separated and housed in cages with one or two littermates of the same gender. They were provided free access to rat chow and water. All groups contained litters of five to 12 pups with similar number of males and females. Extremely large or small litters were eliminated. Offspring were weaned 23 days after birth and group-housed. Male offspring were selected and used for further experiments. A total of four experimental groups of adolescents were tested: 1) control group, offspring of unstressed mothers; 2) PNS group, offspring of mothers subjected to stress before parturition; 3) tianeptine treated group, tianeptine was administered to offspring of mothers subjected to PNS; and 4) haloperidol treated group, haloperidol was administered to offspring of mothers subjected to PNS. For each group, 14 male rats were used.

\section{Behavior Test}

Modified behavioral tests including social interaction test (SIT), open field test (OFT), and forced swim test (FST), were performed as described previously and summarized below. All behavioral tests were commenced at 9 a.m. Rats were allowed to adapt to test environment for at least 30 minutes before behavior test.

\section{SIT}

SIT protocol was adapted from previous studies. ${ }^{7,36,39,40)}$ Test was conducted in a black Polygal chamber $(77 \times 77 \times 25$ $\mathrm{cm}$ ) using rats at 57 days of age. The room in which the chamber was located was darkened during testing. The chamber was illuminated by a single $25 \mathrm{~W}$ red light bulb placed approximately $100 \mathrm{~cm}$ above the base of the chamber. Social interaction partners were same-sex siblings who resided in the same cage after weaning. Their body weights were approximately the same. Each session lasted 20 minutes. Total duration of social play, the number of interaction, and type of interaction were recorded. Behaviors were scored manually by analyzing video tapes. This was achieved by a time-sampling technique performed by an observer blinded to experimental groups. Based on the video, a rater blinded to treatment conditions scored the behavioral activity as aggressive (fighting [kicking, boxing, and wrestling], aggressive grooming, and biting) or non-aggressive (sniffing, following and grooming partner). Experimental and target rats were not used in this test more than once. The arena was cleaned with $70 \%$ ethanol after each trial. ${ }^{40)}$

\section{OFT}

To assess exploratory activity and reactivity to novel environment, OFT was performed as described in previous studies. ${ }^{40-42)}$ Briefly, rats were removed from their home cage on the test day (age, 58 days) and placed individually in the $15 \times 15 \times 20 \mathrm{~cm}$ black Polygal open-field start-box linked to another black polygal OFT box $(77 \times 77 \times 25 \mathrm{~cm})$ for 5 minutes. No background noise was provided. The experimenter exited the room and behaviors of rats in the OFT box were recorded for 20 minutes. Central boxes entered, line crossings, runs, rears, grooming, cage sniff action, and immobile behavior were scored. For each score, frequency and time were recorded.

\section{FST}

FST was performed as described in previous studies. ${ }^{36,41,42)}$ Briefly, rats (age, 59 days) were lowered individually into a cylinder filled with fresh warm tap water $\left(25 \pm 2^{\circ} \mathrm{C}\right)$. Each rat was removed after 15 minutes of test and wiped with a clean towel to remove excess water before being returned to its home cage. Each rat was placed in the cylinder again for 5 minutes on the following day. Swimming, climbing, and immobility behaviors were recorded with a video camera and evaluated by an observer with a stopwatch. Predominant behaviors were counted every 5 seconds. Test scores for swimming activity (horizontal movement throughout the chamber and crossing quadrants), climbing activity (upward movements along the side of the chamber and jump-ups from the bottom of the chamber), and immobility (no additional activity other than keeping the head above water or tiny whip kicks) were recorded as described previously. ${ }^{43,44)}$

\section{Corticosterone Level}

Following behavior tests, adolescent male offspring from each group were moved to the laboratory. On the last behavior test day, experimental subjects were placed 
in cylindrical plastic restraint tubes for 60 minutes ( 5 to 6 animals/group). They were sacrificed by decapitation. Trunk blood was collected immediately into plastic tubes. Blood sample was centrifuged at 13,000 rpm for 10 minutes. Serum was placed in a fresh tube. Brains were removed rapidly from the skull. Each prefrontal cortex was separated, placed in a fresh tube, and frozen in liquid nitrogen. Brain tissue and serum samples were stored at $-80^{\circ} \mathrm{C}$ until use. Serum corticosterone levels were determined by immunoassay using a corticosterone ELISA kit (cat no. 501320; Cayman Chemical, Ann Arbor, MI, USA). Assays were conducted according to the manufacturer's instructions.

\section{Western Blot}

Prefrontal cortical tissues were lysed in RIPA buffer (EBA-1149; Elpis-Biotech, Inc., Daejeon, Korea) containing protease inhibitors and centrifuged at 17,968 $g$ for 10 minutes at $4{ }^{\circ} \mathrm{C}$. To detect Dpys 2 and neurofilament protein, $100 \mu \mathrm{g}$ of lysed proteins were subjected to $10 \%$ and $8 \%$ sodium dodecyl sulfate-polyacrylamide gel electrophoresis (SDS-PAGE) and transferred to polyvinylidene difluoride membrane (Millipore, Milford, MA, USA). After blocking with $5 \%$ skim milk, the polyvinylidene difluoride (PVDF) membranes were probed with anti-Dpys 2 (1:1,000; \#9393; Cell Signaling Technology, Danvers, MA, USA), anti-LIM and SH3 protein 1 (Lasp1, 1:2,000, MAB8991; Millipore), anti-neurofilament $\mathrm{H}$ (NF-H, 1:10,000; ab8135; Abcam, Cambridge, UK), or anti- $\beta$-actin (Actb, 1:1,000; sc-81178; Santa Cruz Biotechnology, Inc., Santa Cruz, CA, USA) antibodies overnight at $4^{\circ} \mathrm{C}$, after which they were incubated with peroxidase-conjugated secondary antibody (1:10,000, N4142; Sigma-Aldrich) for 1 hour at room temperature. Immunoreactive bands were detected using enhanced chemiluminescence kit (Elpis-Biotech, Inc.). Quantitative measurements of Dpysl2, Lasp1, NF-H, and Actb proteins were performed using Image J software $(\mathrm{NIH}$, Bethesda, MD, USA).

\section{Immunohistochemistry}

Rats (4 to 5 animals/group) were deeply anesthetized with ethyl ether and perfused with $4 \%$ paraformaldehyde. Rixed brains were frozen and cut into $30-\mu \mathrm{m}$ sections. Frozen sections from the prefrontal cortex were blocked with normal horse serum (S-2000; Vector Laboratories, Inc., Burlingame, CA, USA), incubated with anti-Dpysl2 (1:1,000, HPA002381; Atlas Antibodies AB, Stockholm, Sweden), anti-NF-H (1:10,000, ab8135; Abcam), or NeuN antibodies (1:100, MAB377; Merck Millipore, Darmstadt, Germany). Sections were incubated with Cy3-conjugated anti-rabbit $(1: 500 ; 715-545-151)$ or anti-mouse (1:800; 111-165-003) secondary antibodies (Jackson ImmunoResearch Laboratories, Inc., West Grove, PA, USA). Fluorescent images were captured using a confocal laser-scanning microscope (FV10-ASW; Olympus, Tokyo, Japan). Images were quantified with Image J software using a protocol described previously ${ }^{43)}$ with slight modification.

\section{Statistical Analyses}

All data are expressed as mean \pm standard error of mean. They were compared using Student's $t$ test and/or ANOVA. If results of ANOVA indicated significant differences, post-hoc analysis was performed with Tukey's honest significant difference (HSD) test. All statistical analyses were performed using IBM SPSS Statistics ver. 19 software (IBM Co., Armonk, NY, USA). Statistical significance was considered at $p<0.05$.

Table 2. Behavior of non-prenatal stress and prenatal stress and tianeptine treatment of prenatal stress in a forced swim test

\begin{tabular}{ccccc}
\hline Forced swim test & NS & PNS & HP & TN \\
\hline Climbing & $12.29 \pm 3.11$ & $5.57 \pm 2.72^{*}$ & $22.71 \pm 3.40^{\dagger}$ & $12.71 \pm 3.58$ \\
Swimming & $35.57 \pm 1.73$ & $32.71 \pm 4.20$ & $27.86 \pm 3.79$ & $36.00 \pm 2.70$ \\
Immobility & $12.14 \pm 2.13$ & $21.71 \pm 2.58^{*}$ & $9.00 \pm 2.20^{\dagger}$ & $11.29 \pm 1.60^{\ddagger}$ \\
\hline
\end{tabular}

Data are presented as mean \pm standard deviation.

PNS, prenatal stressed offspring; NS, non-prenatal stressed offspring; HP, haloperidol treatment with PNS offspring; TN, tianeptine treatment with PNS offspring. $p<0.05$ *between NS and PNS, ${ }^{\dagger}$ between PNS and haloperidol, and ${ }^{\dagger}$ between PNS and TN. 


\section{RESULTS}

\section{FST}

Significant differences in FST results were detected among the four groups (Table 2). PNS offspring exhibited significantly ( $p<0.05$, Tukey's HSD test) increased immobility behaviors compared to rats in the NS group. Decreased climbing function in PNS offspring was significantly ( $p<0.05$, Tukey's HSD test) increased after hal- operidol treatment (Table 2). The increased immobility in PNS offspring was significantly $(p<0.05$, Tukey's HSD test) decreased after treatment with haloperidol or tianeptine (Table 2).

\section{SIT}

The majority of non-aggressive behaviors (sniff, follow, grooming) and aggressive behaviors (fight, aggressive grooming, biting) in SIT (except the number and duration

Table 3. Behavior of non-prenatal stress and prenatal stress and prenatal stress with tianeptine treatment for adolescence in a social interaction test

\begin{tabular}{|c|c|c|c|c|}
\hline Social interaction test & NS & PNS & $\mathrm{HP}$ & $\mathrm{TN}$ \\
\hline \multicolumn{5}{|l|}{ Behavior (n) } \\
\hline Sniff & $66.86 \pm 3.53$ & $27.86 \pm 2.11^{*}$ & $73.00 \pm 3.48^{\dagger}$ & $64.43 \pm 5.08^{\ddagger}$ \\
\hline Follow & $17.57 \pm 1.00$ & $1.14 \pm 0.46^{*}$ & $6.00 \pm 1.65^{\dagger}$ & $3.14 \pm 0.91$ \\
\hline Grooming the partner & $8.14 \pm 0.86$ & $1.86 \pm 0.88^{*}$ & $10.86 \pm 1.26^{\dagger}$ & $8.57 \pm 1.54^{\ddagger}$ \\
\hline Fight & $2.86 \pm 0.94$ & $4.28 \pm 0.74$ & $0.00 \pm 0.00^{\dagger}$ & $0.00 \pm 0.00^{\ddagger}$ \\
\hline Aggressive grooming & $0.43 \pm 0.20$ & $8.00 \pm 1.23^{*}$ & $0.29 \pm 0.29^{\dagger}$ & $0.14 \pm 0.14^{\ddagger}$ \\
\hline Biting & $0.43 \pm 0.30$ & $1.71 \pm 0.68$ & $0.29 \pm 0.18$ & $0.14 \pm 0.14^{\ddagger}$ \\
\hline \multicolumn{5}{|l|}{ Duration (sec) } \\
\hline Sniff & $111.57 \pm 6.69$ & $85.57 \pm 4.88^{*}$ & $122.29 \pm 3.96^{\dagger}$ & $99.43 \pm 3.64^{\ddagger}$ \\
\hline Follow & $26.71 \pm 2.09$ & $1.71 \pm 0.71^{*}$ & $7.57 \pm 1.78^{\dagger}$ & $4.86 \pm 1.35$ \\
\hline Grooming the partner & $19.14 \pm 1.45$ & $3.00 \pm 1.46^{*}$ & $31.00 \pm 2.84^{\dagger}$ & $28.43 \pm 2.93^{\ddagger}$ \\
\hline Fight & $10.24 \pm 4.61$ & $12.14 \pm 4.73$ & $0.00 \pm 0.00^{\dagger}$ & $0.00 \pm 0.00^{\ddagger}$ \\
\hline Aggressive grooming & $0.71 \pm 0.36$ & $13.00 \pm 2.89 *$ & $0.71 \pm 0.71^{\dagger}$ & $0.43 \pm 0.43^{\ddagger}$ \\
\hline Biting & $0.43 \pm 0.30$ & $3.71 \pm 1.36^{*}$ & $0.29 \pm 0.18^{\dagger}$ & $0.14 \pm 0.14^{\ddagger}$ \\
\hline
\end{tabular}

Data are presented as mean \pm standard error of mean.

PNS, prenatal stressed offspring; NS, non-prenatal stressed offspring; HP, haloperidol treatment with PNS offspring; TN, tianeptine treatment with PNS offspring.

$p<0.05$ *between NS and PNS, ${ }^{\dagger}$ between PNS and haloperidol, and ${ }^{\ddagger}$ between PNS and TN.

Table 4. Behavior of non-prenatal stress and prenatal stress and prenatal stress with tianeptine treatment for adolescence in an open field test

\begin{tabular}{|c|c|c|c|c|}
\hline Open field test & NS & PNS & $\mathrm{HP}$ & $\mathrm{TN}$ \\
\hline \multicolumn{5}{|l|}{ Behavior (n) } \\
\hline Central entered & $18.29 \pm 2.18$ & $7.00 \pm 1.36^{*}$ & $19.86 \pm 1.82^{\dagger}$ & $16.14 \pm 1.84^{\ddagger}$ \\
\hline Line crossing & $7.29 \pm 1.51$ & $1.86 \pm 0.59^{*}$ & $9.86 \pm 0.86^{\dagger}$ & $8.14 \pm 1.60^{\ddagger}$ \\
\hline Run & $3.57 \pm 2.16$ & $3.86 \pm 0.94$ & $5.14 \pm 1.72$ & $12.57 \pm 4.71$ \\
\hline Rear & $84.86 \pm 7.60$ & $48.86 \pm 7.97^{*}$ & $87.14 \pm 4.47^{\dagger}$ & $83.71 \pm 11.24^{\ddagger}$ \\
\hline Grooming & $11.00 \pm 1.83$ & $12.29 \pm 1.25$ & $17.14 \pm 1.88$ & $15.29 \pm 3.59$ \\
\hline Cage sniff & $115.00 \pm 7.90$ & $80.86 \pm 15.19$ & $114.86 \pm 5.01$ & $140.71 \pm 6.57^{\ddagger}$ \\
\hline Immobility & $0.14 \pm 0.14$ & $3.00 \pm 1.11^{*}$ & $0.00 \pm 0.00^{\dagger}$ & $0.00 \pm 0.00^{\ddagger}$ \\
\hline \multicolumn{5}{|l|}{ Duration (sec) } \\
\hline Run & $3.86 \pm 2.27$ & $5.71 \pm 1.63$ & $5.57 \pm 1.97$ & $14.00 \pm 5.45$ \\
\hline Rear & $170.57 \pm 19.15$ & $100.71 \pm 13.93^{*}$ & $174.29 \pm 6.81^{\dagger}$ & $156.43 \pm 21.48^{\ddagger}$ \\
\hline Grooming & $143.57 \pm 34.65$ & $182.14 \pm 26.27$ & $204.29 \pm 28.42$ & $179.71 \pm 46.46$ \\
\hline Cage sniff & $406.29 \pm 27.92$ & $262.71 \pm 43.02^{*}$ & $391.00 \pm 37.06^{\dagger}$ & $419.14 \pm 37.23^{\ddagger}$ \\
\hline Immobility & $4.14 \pm 4.14$ & $20.14 \pm 11.52$ & $0.00 \pm 0.00$ & $0.00 \pm 0.00$ \\
\hline
\end{tabular}

Data are presented as mean \pm standard error of mean.

PNS, prenatal stressed offspring; NS, non-prenatal stressed offspring; HP, haloperidol treatment with PNS offspring; TN, tianeptine treatment with PNS offspring. $p<0.05$ * between NS and PNS, ${ }^{\dagger}$ between PNS and haloperidol, and ${ }^{\ddagger}$ between PNS and TN. 
of fights or the number of biting) were changed significantly ( $p<0.05$, Tukey's HSD test) in the PNS group. However, these changes were restored ( $p<0.05$, Tukey's HSD test) by treatment with haloperidol or tianeptine (Table 3).

\section{OFT}

NS and PNS offspring were subjected to OFT for 20 minutes. The PNS group showed significant changes in the number of central entries, line crossings, number and duration of rear, duration of cage sniff, and the number of immobility compared to the NS group. These changes were restored ( $p<0.05$, Tukey's HSD test) after treatment with haloperidol or tianeptine (Table 4). The PNS group showed significantly decreases in the number of central entries, line-crossing, number/duration of rear behaviors, and duration of cage sniff compared to the NS group. However, these scores were increased ( $p<0.05$, Tukey's HSD test) after treatment with haloperidol or tianeptine. On the other hand, the number of immobility behaviors was increased in the PNS group compared to that in the NS group. After treatment with haloperidol or tianeptine, it was reverted ( $p<0.05$, Tukey's HSD test) to control levels (Table 4).

\section{Western Blot and Immunohistochemistry}

During development, neurons of the central nervous system express Dpys/2 that which plays a key role in axon
A


B



D

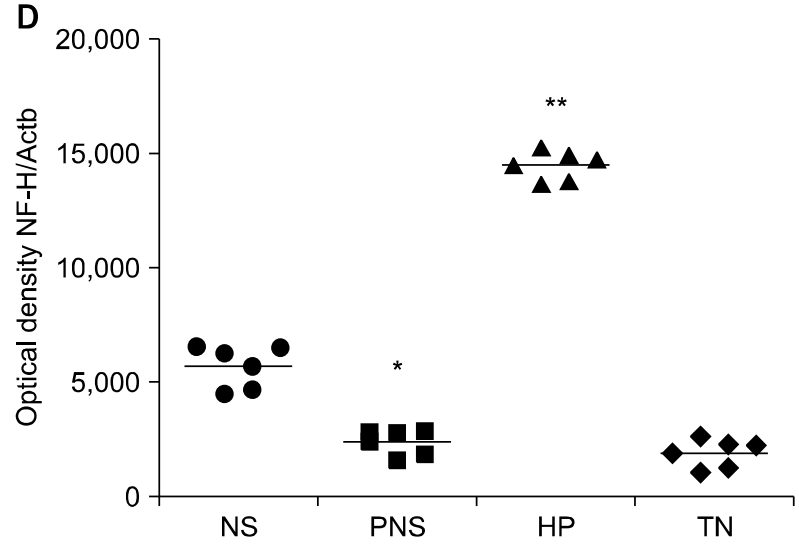

Fig. 1. Western blot analysis of Dpysl2, Lasp1, and NF-H expression in brains of prenatal stress induced rats. (A) Dpysl2, Lasp1, and NF-H protein expression by Western blotting using Actb as an internal control. PNS rats demonstrated decreased expression of Dpys/2, Lasp1, and NF-H in the frontal cortex. Decreased expression levels of Dpysl2 and Lasp1 were recovered by tianeptine treatment. (B) Scatter gram in the graph indicating standard error mean (SEM) ( $p<0.05$ compared to the prenatal stress group in Lasp1 expression). (C) Scatter gram in the graph indicating SEM ( $p<0.05$ compared to prenatal stress group in DpysI 2 expression). (D) Scatter gram in the graph indicating SEM ( $p<0.05$ compared to prenatal stress group in NF-H expression).

NS, non-prenatal stressed offspring; PNS, prenatal stressed offspring; HP, haloperidol treatment offspring; TN, tianeptine treatment offspring. *NS and PNS, **PNS and HP, ***PNS and TN.

Data are presented in mean $\pm \mathrm{SEM} ; p<0.05$. 
A



B

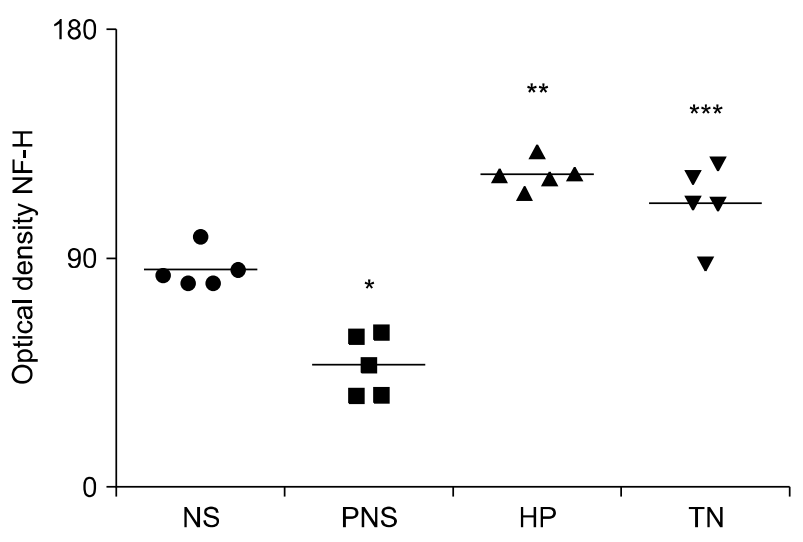

Fig. 2. Immunohistochemical analysis of NF-H expression in brains of prenatal stress induced rats. (A) Confocal microscopic image showing immunofluorescent staining for $\mathrm{NF}-\mathrm{H}$ (anti-NF-H, red, Cy3) with NeuN in the frontal cortex. Fluorescent staining revealed decreased NF-H in these regions. Scale bar, FC: $50 \mu \mathrm{m}$. (B) Scatter gram in the graph indicating standard error of mean (SEM) $(p<0.05$ compared to the prenatal stress group in the frontal cortex).

FC, frontal cortex; NS, non-prenatal stressed offspring; PNS, prenatal stressed offspring; HP, haloperidol treatment offspring; TN, tianeptine treatment offspring.

*NS and PNS, **PNS and HP, ***PNS and TN.

Data are presented in mean \pm SEM; $p<0.05$.

formation concentrated at the synaptic sites. It also affects synaptic physiology in axons. ${ }^{45)}$ In our previous report, Dpys 2 levels have been found to be decreased in the prefrontal cortex of PNS offspring rats. ${ }^{17)}$ To determine the effect of PNS on neurodevelopmental proteins Lasp1, Dpysl2, and NF-H, we performed Western blots (Fig. 1) and immunohistochemical analyses (Figs. 2, 3) for prefrontal cortex areas from brains of NS, PNS, haloperidol-, or tianeptine-treated offspring. In the prefrontal cortex, levels of these proteins were significantly lower in PNS than those in NS rats (all $p<0.05$, Tukey's HSD test; Fig. 1). Expression levels of the protein were altered by haloperidol or tianeptine treatment. Despite these increases, we were unable to conclude that such increases were directly induced by haloperidol or tianeptine because drug treated NS groups were not included in this study. However, it was evident that haloperidol and tianeptine affected protein expression levels, at least partially. To confirm the expression of these proteins, we also per- formed immunohistochemical staining using brain tissues. Results revealed significant increases of these proteins (all $p<0.05$, Tukey's HSD test; Fig. 1). Differentially expressed protein levels of Dpysl2 and NF-H were also evident in immunofluorescent-stained brain images among the four groups. Their intensities in immunohistochemical staining showed significant differences $(p<0.05$, Tukey's HSD test; Figs. 2, 3).

\section{Corticosterone Level}

Increased stress-induced corticosterone levels were evident in the PNS group compared to those in the NS group. Corticosterone levels in haloperidol or tianeptine treated group were significantly (both $p<0.05$, Tukey's HSD test) decreased to levels in the NS group (Fig. 4).

\section{DISCUSSION}

In this study, behavioral and protein expression analy- 
A

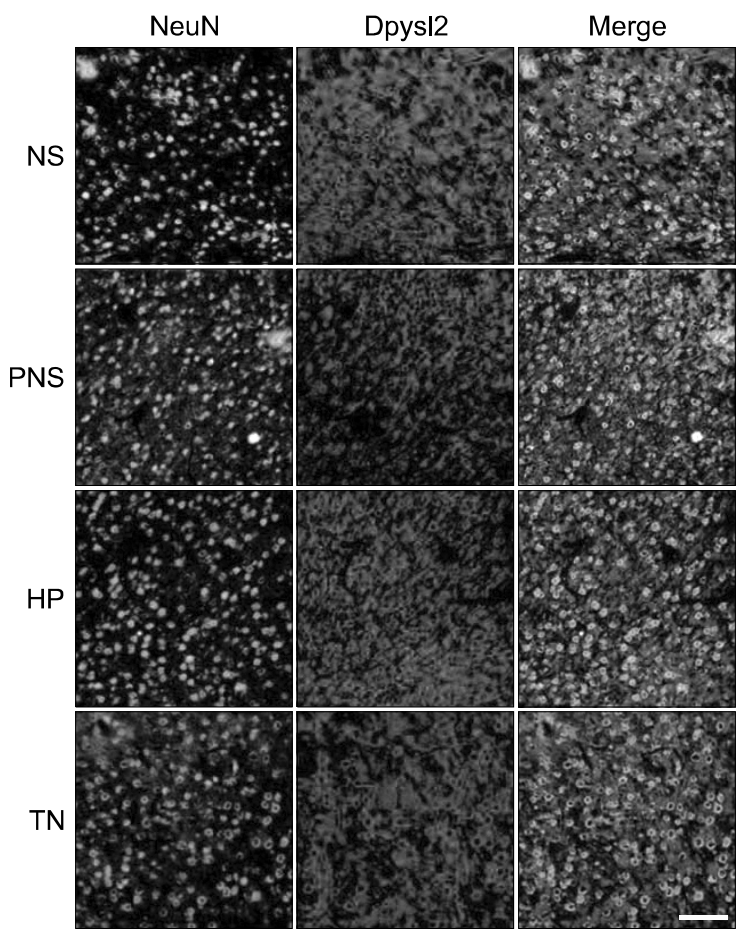

B

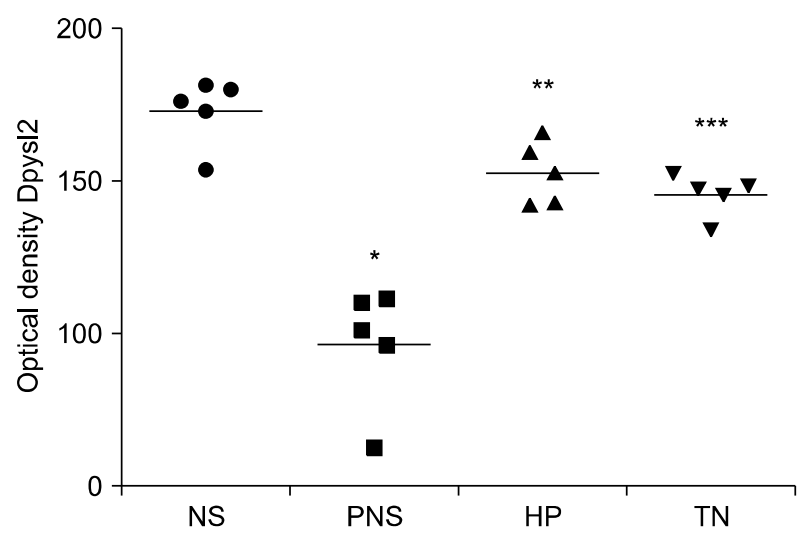

Fig. 3. Immunohistochemical analysis of Dpysl2 expression in brains of prenatal stress induced rats. (A) Confocal microscopic image showing immunofluorescent staining for Dpysl2 (anti-Dpysl2, red, Cy3) with NeuN in the frontal cortex. Fluorescent staining revealed decreased Dpysl2 in these regions. Scale bar, FC; $50 \mu \mathrm{m}$. (B) Scatter gram in the graph indicating standard error mean (SEM) $(p<0.05$ compared to prenatal stress group in the frontal cortex).

NS, non-prenatal stressed offspring; PNS, prenatal stressed offspring; HP, haloperidol treatment offspring; TN, tianeptine treatment offspring. *NS and PNS, **PNS and HP, ***PNS and TN.

Data are presented in mean $\pm \mathrm{SEM} ; p<0.05$.

ses were performed in a PNS animal model to examine the preventive effect of antidepressants on stress-related psychiatric disorders such as depression and schizophrenia according to neurodevelopmental theory.

Although tianeptine has unique pharmacological properties, its structure is related to tricyclic agents, likely other antidepressants. ${ }^{46)}$ Therapeutic effects of tianeptine do not immediately modulate monoamine axes. However, its effect can trigger the modulation of glutamatergic signaling associated with a cascade of cellular adaptations, which can ultimately result in antidepressant actions. ${ }^{25,31)}$ Tianeptine is particularly effective in improving stress-induced cognitive defects and preventing structural and functional impairments in cortical and limbic regions. ${ }^{24,31,477}$ It has been reported that tianeptine treatment can reduce the immobility time of PNS rats in FST. ${ }^{34)}$ Authors in that study included only restraint stress in the PNS paradigm for depression-like animal model. However, we used PNS model as a neurodevelopmental model for schizophrenia

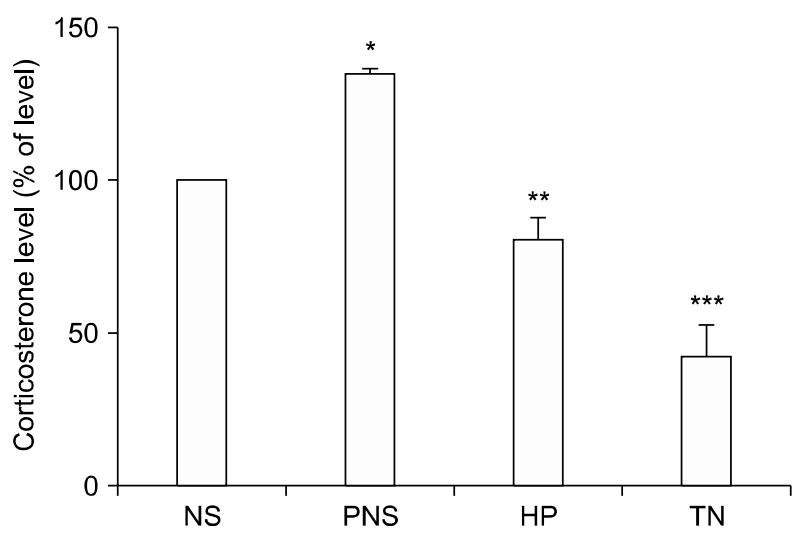

Fig. 4. Percent of corticosterone levels in adolescent male rats. These were analyzed for male offspring of rats exposed to stress during gestation in the last behavior test or male rats derived from mothers without stress during gestation. Corticosterone secretion was induced by holding rats in restraint tubes for 60 minutes. Corticosterone levels were increased in the PNS group but decreased in haloperidol or tianeptine treatment groups.

NS, non-prenatal stressed offspring; PNS, prenatal stressed offspring; $\mathrm{HP}$, haloperidol treatment offspring; $\mathrm{TN}$, tianeptine treatment offspring.

${ }^{* N S}$ and PNS, **PNS and $\mathrm{HP},{ }^{* * *} \mathrm{PNS}$ and TN.

Data are presented in mean \pm standard error mean; $p<0.05$. 
and included repeated and variable stress paradigm. ${ }^{10)}$ Tianeptine treatment reduced FST immobility time in our study. Furthermore, this treatment restored abnormal number of central entries, line-crossing, number/duration of rear behaviors, duration of cage sniff, and the number of immobility in OFT. Notably, tianeptine also reduced aggressive behavior and increased non-aggressive behavior during SIT in this study.

In this manuscript, we presented therapeutic effects of an antidepressant, tianeptine, and an antipsychotic, haloperidol, in PNS model. Tianeptine works by decreasing extracellular levels of serotonin in rat brain without decreasing serotonin release, ${ }^{24)}$ while haloperidol works by blocking the effect of dopamine and increasing its turnover rate. ${ }^{48)}$ Although the action mechanisms of the two drugs are different, Western blot and immunohistochemical analyses of the prefrontal cortex revealed that the downregulation of several neurodevelopmental proteins in PNS rats was restored after treatment with tianeptine or haloperidol. This might explain why behavioral changes in PNS rats were restored after treatment with tianeptine or haloperidol. In this study, we focused on the effect of the two drugs on the recovery of neurodevelopmental deficit induced by PNS without focusing on the exact mechanisms involved. Haloperidol was used as a positive control as an antipsychotic drug based on previous studies. Therefore, the exact reason why effects of tianeptine and haloperidol on PNS rats are similar is currently unclear. Several studies have shown that the effect of antipsychotic drug haloperidol on dendritic architecture is at the developmental stage in animal models, corresponding to fetal or fetal plus early childhood stages in human. ${ }^{27)}$ Chronic haloperidol treatment during early postnatal life induces persistent alterations in motor and associative-spatial systems. ${ }^{49)}$ It also affects the expression levels of central neurotransmitters and receptors in rats. Furthermore, the effect of haloperidol depends on the stage of development when it is administered. It can induce some behavioral activity and neurochemical and molecular alterations. ${ }^{49)}$ Contrarily, several studies have reported that this neuroleptic treatment has side effects with higher rates of nonresponse in children and adolescents compared to adults. ${ }^{50,51)}$ However, some studies have shown that haloperidol is effective in adolescents and early adults. ${ }^{52-54)}$ Previous studies have shown that adolescent male rats are highly sensitive to haloper- idol-induced suppression of locomotor activity, more than adult male rats. ${ }^{52-54)}$ These results highlights the importance of early intervention for psychotic symptoms and depression of schizophrenia. ${ }^{55,56)}$

The common feature of PNS models is that critical experimental manipulation can be made to interrupt early brain and central nervous system development, thus inducing significant neurodevelopmental changes, increasing maternal stress hormones, and changing responses to prenatal stressors. ${ }^{2,57)}$ Impaired social interaction behavior was observed in prepubertal PNS rats at age of 56 days and in young adult PNS rats. It has been reported that social withdrawal of adolescence period is associated with the first clinical sign of human schizophrenia. ${ }^{39,57,58)}$ Therefore, social withdrawal in PNS rats might be related to schizophrenia symptoms, further supporting the relevance of this model to the schizophrenia phenotype. Such diminution in social interaction behavior reflects increased anxiety in PNS rats. ${ }^{3)}$ Neurofilaments form part of the axon skeleton and functionally maintain neuronal caliber might also play a role in intracellular transport to axons and dendrites. ${ }^{59)}$ It has been found that these subunits are changed in schizophrenia and mood disorder based on distribution and expression studies of pyramidal neurons in prefrontal cortex. ${ }^{60)}$

In the present report, we also investigated the expression levels of two neurodevelopment-related proteins based on previous reports. ${ }^{17,61)}$ Our results revealed that application of repeated and variable PNS paradigm during critical periods of fetal brain development could result in changes in the expression of neurodevelopmental proteins, such as neurofilament proteins and Dpysl2. These proteins might have enduring effects on axonal outgrowth and synaptic function in the offspring later during adulthood. ${ }^{40,58)}$ Decrease in protein expression of NF-H and Dpys 2 due to PNS was affected by haloperidol or tianeptine treatment. Therefore, these proteins might be involved in behavioral abnormalities of PNS rats directly and/or indirectly.

The present results indicate an additional role of antidepressant tianeptine in the pathogenesis of psychiatric disorders like schizophrenia. Many studies have focused on the effect of antipsychotics such as haloperidol in prenatal period and its side effects in adolescents or early adults. In contrast, we investigated the effect of tianeptine in a model geared toward adolescents and early adults by 
analyzing expression levels of neurodevelopment proteins and alterations in behavioral patterns. Further research studies using cellular and animal model systems are required to characterize pharmacological functions and signal transduction pathways of drugs in this schizophrenia-like animal model.

\section{Acknowledgments}

The present study was performed with the support of Basic Science Research Program through the National Research Foundation of Korea (NRF) funded by the Ministry of Education. (2016R1D1A1B03931619) and 'Cooperative Research Program for Agriculture Science and Technology Development (project no. PJ011582)' of the Rural Development Administration, Korea.

\section{REFERENCES}

1. Imamura $\mathrm{Y}, \mathrm{Nakane} \mathrm{Y}$, Ohta $\mathrm{Y}, \mathrm{Kondo} \mathrm{H}$. Lifetime prevalence of schizophrenia among individuals prenatally exposed to atomic bomb radiation in Nagasaki City. Acta Psychiatr Scand 1999; 100:344-349.

2. Meyer U, Feldon J. Epidemiology-driven neurodevelopmental animal models of schizophrenia. Prog Neurobiol 2010;90:285-326.

3. Weinstock M. The long-term behavioural consequences of prenatal stress. Neurosci Biobehav Rev 2008;32:1073-1086.

4. Seckl JR. Prenatal g/ucocorticoids and long-term programming. Eur J Endocrinol 2004;151 Supp/ 3:U49-U62.

5. de Kloet ER, Sibug RM, Helmerhorst FM, Schmidt MV. Stress, genes and the mechanism of programming the brain for later life. Neurosci Biobehav Rev 2005;29:271-281.

6. Beydoun H, Saftlas AF. Physical and mental health outcomes of prenatal maternal stress in human and animal studies: a review of recent evidence. Paediatr Perinat Epidemiol 2008;22: 438-466.

7. Kuperberg G, Heckers S. Schizophrenia and cognitive function. Curr Opin Neurobiol 2000;10:205-210.

8. Kinnunen AK, Koenig JI, Bilbe G. Repeated variable prenatal stress alters pre- and postsynaptic gene expression in the rat frontal pole. I Neurochem 2003;86:736-748.

9. Koenig JI, Elmer GI, Shepard PD, Lee PR, Mayo C, Joy B, et al. Stress during gestation produces alterations in adult rat behavior: relevance to schizophrenia. Program No. 495.06. 2002 Neuroscience Meeting Planner. Orlando, FL:Society for Neuroscience;2002.

10. Koenig JI, Elmer GI, Shepard PD, Lee PR, Mayo C, Joy B, et al. Prenatal exposure to a repeated variable stress paradigm elicits behavioral and neuroendocrinological changes in the adult offspring: potential relevance to schizophrenia. Behav Brain
Res 2005; 156:251-261.

11. Lemaire V, Koehl M, Le Moal M, Abrous DN. Prenatal stress produces learning deficits associated with an inhibition of neurogenesis in the hippocampus. Proc Natl Acad Sci U S A 2000;97:11032-11037.

12. Martínez-Téllez RI, Hernández-Torres E, Gamboa C, Flores G. Prenatal stress alters spine density and dendritic length of nucleus accumbens and hippocampus neurons in rat offspring. Synapse 2009;63:794-804.

13. Koehl M, Barbazanges A, Le Moal M, Maccari S. Prenatal stress induces a phase advance of circadian corticosterone rhythm in adult rats which is prevented by postnatal stress. Brain Res 1997;759:317-320.

14. Maccari S, Piazza PV, Kabbaj M, Barbazanges A, Simon H, Le Moal M. Adoption reverses the long-term impairment in g/ucocorticoid feedback induced by prenatal stress. J Neurosci 1995; 15:110-116.

15. Van den Hove DL, Kenis G, Brass A, Opstelten R, Rutten BP, Bruschettini $M$, et al. Vulnerability versus resilience to prenatal stress in male and female rats; implications from gene expression profiles in the hippocampus and frontal cortex. Eur Neuropsychopharmacol 2013;23:1226-1246.

16. Mairesse J, Vercoutter-Edouart AS, Marrocco J, Zuena AR, Giovine A, Nicoletti F, et al. Proteomic characterization in the hippocampus of prenatally stressed rats. J Proteomics 2012; 75:1764-1770.

17. Lee H, Joo J, Nah SS, Kim JW, Kim HK, Kwon JT, et al. Changes in Dpys/2 expression are associated with prenatally stressed rat offspring and susceptibility to schizophrenia in humans. Int J Mol Med 2015;35:1574-1586.

18. Carlezon WA Jr, Duman RS, Nestler EJ. The many faces of CREB. Trends Neurosci 2005;28:436-445.

19. Nibuya M, Nestler EJ, Duman RS. Chronic antidepressant administration increases the expression of CAMP response element binding protein (CREB) in rat hippocampus. J NeurosCi 1996; 16:2365-2372.

20. Thome J, Sakai N, Shin K, Steffen C, Zhang YJ, Impey S, et al. cAMP response element-mediated gene transcription is upregulated by chronic antidepressant treatment. / Neurosci 2000;20:4030-4036.

21. Kuipers E, Garety P, Fowler D, Freeman D, Dunn G, Bebbington P. Cognitive, emotional, and social processes in psychosis: refining cognitive behavioral therapy for persistent positive symptoms. Schizophr Bull 2006;32 Suppl 1:S24-S31.

22. Magariños AM, Deslandes A, McEwen BS. Effects of antidepressants and benzodiazepine treatments on the dendritic structure of CA3 pyramidal neurons after chronic stress. Eur J Pharmacol 1999;371:113-122.

23. Weinstock M. Alterations induced by gestational stress in brain morphology and behaviour of the offspring. Prog Neurobiol 2001;65:427-451.

24. Czéh B, Michaelis T, Watanabe T, Frahm J, de Biurrun G, van Kampen M, et al. Stress-induced changes in cerebral metabo- 
lites, hippocampal volume, and cell proliferation are prevented by antidepressant treatment with tianeptine. Proc Natl Acad Sci U S A 2001;98:12796-12801.

25. Zoladz JA, Pilc A, Majerczak J, Grandys M, Zapart-Bukowska J, Duda K. Endurance training increases plasma brain-derived neurotrophic factor concentration in young healthy men. J Physiol Pharmacol 2008;59 Supp/ 7:119-132.

26. McEwen BS, Chattarji S, Diamond DM, Jay TM, Reagan LP, Svenningsson $\mathrm{P}$, et al. The neurobiological properties of tianeptine (Stablon): from monoamine hypothesis to glutamatergic modulation. Mol Psychiatry 2010;15:237-249.

27. Song JC, Seo MK, Park SW, Lee JG, Kim YH. Differential effects of olanzapine and haloperidol on MK-801-induced memory impairment in mice. Clin Psychopharmacol Neurosci 2016;14:279-285.

28. Stone JM. Glutamatergic antipsychotic drugs: a new dawn in the treatment of schizophrenia? Ther Adv Psychopharmacol 2011;1:5-18.

29. Paul IA, Skolnick P. Glutamate and depression: clinical and preclinical studies. Ann N Y Acad Sci 2003; 1003:250-272.

30. Zarate CA Jr, Du J, Quiroz J, Gray NA, Denicoff KD, Singh J, et al. Regulation of cellular plasticity cascades in the pathophysiology and treatment of mood disorders: role of the glutamatergic system. Ann N Y Acad Sci 2003;1003:273-291.

31. McEwen BS, Gianaros PJ. Central role of the brain in stress and adaptation: links to socioeconomic status, health, and disease. Ann N Y Acad Sci 2010;1186:190-222.

32. Kuipers SD, Trentani A, van der Zee EA, den Boer JA. Chronic stress-induced changes in the rat brain: role of sex differences and effects of long-term tianeptine treatment. Neuropharmacology 2013;75:426-436.

33. Della FP, Abelaira HM, Réus GZ, Ribeiro KF, Antunes AR, Scaini $\mathrm{G}$, et al. Tianeptine treatment induces antidepressivelike effects and alters BDNF and energy metabolism in the brain of rats. Behav Brain Res 2012;233:526-535.

34. Morley-Fletcher S, Darnaudery M, Koehl M, Casolini P, Van Reeth O, Maccari S. Prenatal stress in rats predicts immobility behavior in the forced swim test. Effects of a chronic treatment with tianeptine. Brain Res 2003;989:246-251.

35. Minabe Y, Watanabe K, Nishimura T, Ashby CR Jr. Acute and chronic administration of clozapine produces greater proconvulsant actions than haloperidol on focal hippocampal seizures in freely moving rats. Synapse 1998;29:272-278.

36. Iñiguez SD, Alcantara LF, Warren BL, Riggs LM, Parise EM, Vialou $\mathrm{V}$, et al. Fluoxetine exposure during adolescence alters responses to aversive stimuli in adulthood. I Neurosci 2014; 34:1007-1021.

37. National Research Council. Guide for the care and use of laboratory animals. Washington, DC:National Academy Press; 1996.

38. Charil A, Laplante DP, Vaillancourt C, King S. Prenatal stress and brain development. Brain Res Rev 2010;65:56-79.

39. Campbell A, Baldessarini RJ. Effects of maturation and aging on behavioral responses to haloperidol in the rat. Psychopharmacology (Berl) 1981;73:219-222.

40. Kim YO, Lee HY, Won H, Nah SS, Lee HY, Kim HK, et al. Influence of Panax ginseng on the offspring of adult rats exposed to prenatal stress. Int J Mol Med 2015;35:103-109.

41. Cornblatt BA. The New York high risk project to the Hillside recognition and prevention (RAP) program. Am J Med Genet 2002;114:956-966.

42. Becker A, Peters B, Schroeder H, Mann T, Huether G, Grecksch G. Ketamine-induced changes in rat behaviour: a possible animal model of schizophrenia. Prog Neuropsychopharmacol Biol Psychiatry 2003;27:687-700.

43. Dulawa SC, Holick KA, Gundersen B, Hen R. Effects of chronic fluoxetine in animal models of anxiety and depression. Neuropsychopharmacology 2004;29:1321-1330.

44. Schroeder M, Sultany T, Weller A. Prenatal stress effects on emotion regulation differ by genotype and sex in prepubertal rats. Dev Psychobiol 2013;55:176-192.

45. Goshima Y, Nakamura F, Strittmatter P, Strittmatter SM. Collapsin-induced growth cone collapse mediated by an intracellular protein related to UNC-33. Nature 1995; 376:509514.

46. Kuipers SD, Trentani A, van der Zee EA, den Boer JA. Chronic stress-induced changes in the rat brain: role of sex differences and effects of long-term tianeptine treatment. Neuropharmacology 2013;75:426-436.

47. Reagan LP, Rosell DR, Wood GE, Spedding M, Muñoz C, Rothstein J, et al. Chronic restraint stress up-regulates GLT-1 mRNA and protein expression in the rat hippocampus: reversal by tianeptine. Proc Natl Acad Sci U S A 2004;101: 2179-2184.

48. Bluth R, Langnickel R. Effects of haloperidol and low dose clozapine on the acetylcholine turnover rate in rat forebrain structures. Biomed Biochim Acta 1985;44:1531-1539.

49. Wolansky MJ, Soiza-Reilly M, Fossati M, Azcurra JM. Postnatal haloperidol eliminates the deficit in circling behavior produced by prenatal exposure to the same drug. Neurotoxicol Teratol 2004;26:561-569.

50. Remschmidt H, Hennighausen K, Clement HW, Heiser P, Schulz E. Atypical neuroleptics in child and adolescent psychiatry. Eur Child Adolesc Psychiatry 2000;9 Suppl 1: 19-19.

51. Toren $\mathrm{P}$, Laor N, Weizman A. Use of atypical neuroleptics in child and adolescent psychiatry. J Clin Psychiatry 1998;59: 644-656.

52. Wiley JL. Antipsychotic-induced suppression of locomotion in juvenile, adolescent and adult rats. Eur I Pharmacol 2008; 578:216-221.

53. Campbell A, Baldessarini RJ, Teicher MH. Decreasing sensitivity to neuroleptic agents in developing rats; evidence for a pharmacodynamic factor. Psychopharmacology (Berl) 1988; 94:46-51.

54. DeQuardo JR. Pharmacologic treatment of first-episode schiz- 
ophrenia: early intervention is key to outcome. JClin Psychiatry 1998;59 Supp/ 19:9-17.

55. Stafford MR, Mayo-Wilson E, Loucas CE, James A, Hollis C, Birchwood $\mathrm{M}$, et al. Efficacy and safety of pharmacological and psychological interventions for the treatment of psychosis and schizophrenia in children, adolescents and young adults: a systematic review and meta-analysis. PLoS One 2015;10: e0117166.

56. Kelley ME, Gilbertson M, Mouton A, van Kammen DP. Deterioration in premorbid functioning in schizophrenia: a developmental model of negative symptoms in drug-free patients. Am J Psychiatry 1992;149:1543-1548.

57. Møller P, Husby R. The initial prodrome in schizophrenia: searching for naturalistic core dimensions of experience and behavior. Schizophr Bull 2000;26:217-232.

58. Lee $\mathrm{H}$, Won $\mathrm{H}, \mathrm{Im} \mathrm{J}$, Kim YO, Lee $\mathrm{S}, \mathrm{Cho} \mathrm{IH}$, et al. Effect of
Valeriana fauriei extract on the offspring of adult rats exposed to prenatal stress. Int J Mol Med 2016;38:251-258.

59. Cassereau J, Nicolas G, Lonchampt $\mathrm{P}$, Pinier M, Barthelaix A, Eyer J, et al. Axonal regeneration is compromised in NFH$\mathrm{LaCZ}$ transgenic mice but not in NFH-GFP mice. Neuroscience 2013;228:101-108.

60. Law AJ, Harrison PJ. The distribution and morphology of prefrontal cortex pyramidal neurons identified using anti-neurofilament antibodies SMI32, N200 and FNP7. Normative data and a comparison in subjects with schizophrenia, bipolar disorder or major depression. J Psychiatr Res 2003;37:487-499.

61. Joo J, Lee S, Nah SS, Kim YO, Kim DS, Shim SH, et al. Lasp1 is down-regulated in $\mathrm{NMDA}$ receptor antagonist-treated mice and implicated in human schizophrenia susceptibility. I Psychiatr Res 2013;47:105-112. 